\title{
Cytometric evaluation of transferrin receptor 1 (CD71) in childhood acute lymphoblastic leukemia
}

\author{
Anna Płoszyńska ${ }^{*}$, Katarzyna Ruckemann-Dziurdzińska ${ }^{2 *}$, Agnieszka Jóźwik², \\ Anna Mikosik², Katarzyna Lisowska², Anna Balcerska', Jacek M. Witkowski \\ ${ }^{1}$ Department of Pediatrics, Hematology, Oncology and Endocrinology, \\ Medical University of Gdansk, Poland \\ ${ }^{2}$ Department of Pathophysiology, Medical University of Gdansk, Poland \\ *Equal contribution of both authors
}

\begin{abstract}
Transferrin receptor 1 (CD71) is a transmembrane glycoprotein responsible for cellular iron uptake. Higher expression of CD71 has been identified as a negative prognostic marker for numerous solid tumor types and for some lymphomas. The aim of this study was to evaluate CD71 expression on acute lymphoblastic leukemia (ALL) cells and to follow its possible clinical correlations. Sixty one patients, aged 1-17 years and diagnosed with ALL, were enrolled in the study. CD71 expression was analyzed on the bone marrow blastic cells by flow cytometry. CD71 expression on the leukemic blasts was diversified; in most patients, all blastic cells showed expression of CD71, but levels of expression varied. CD71 expression was statistically higher on T-lineage leukemias. Within the B lineage ALL, a significant difference in CD71 expression existed between precursor B ALL and mature B-ALL, which showed higher CD71 expression. CD71 expression positively correlated with Hgb concentration at diagnosis. Initial risk group assessment and therapy response were not correlated with CD71 expression, although disease free and overall survival times tended to be shorter in patients with B-lineage leukemias with initial high CD71 expression. (Folia Histochemica et Cytobiologica 2012, Vol. 50, No. 2, 304-311)
\end{abstract}

Key words: transferrin receptor $1, \mathrm{CD} 71$, acute lymphoblastic leukemia

\section{Introduction}

The transferrin receptor 1 (TfR1), designated CD71, is a type II transmembrane homodimeric glycoprotein $(180 \mathrm{kDa})$ responsible mainly for cellular iron uptake via internalization of iron loaded transferrin $[1,2]$. This makes iron available for heme synthesis and for many metabolic processes necessary for cell growth and proliferation, including DNA synthesis, electron transport, nitrogen fixation and oxygen sensing $[1,2]$.

\footnotetext{
Correspondence address: K. Ruckemann-Dziurdzińska, Department of Pathophysiology,

Medical University of Gdansk,

Dębinki Str. 7, 80-211 Gdansk, Poland;

tel./fax: + 485834915 10;

e-mail:kruck@gumed.edu.pl
}

CD71 is expressed at high levels on cells with high iron demand, such as maturing erythroid cells and placental trophoblast cells, and on cells with a high proliferation rate, such as cells of the basal epidermis and intestinal epithelium [2-4]. Activated peripheral blood mononuclear cells express high levels of TfR [2]. This may be attributable to the increased need for iron as a cofactor of ribonucleotide reductase, which activity supplies substrates for DNA synthesis [5]. CD71 overexpression has been reported in many neoplasms, including solid tumors such as lung [6,7], bladder [8], colon [9], pancreas [10] and breast cancer [11, 12], gliomas [13] as well as hematological malignancies [5, 14-16]. Higher expression of CD71 has been identified as a negative prognostic marker for numerous solid tumor types $[10,12]$ as well as for non-Hodgkin's lymphoma $[4,17$, 18]. Antibodies targeting CD71 have been introduced as an experimental adjuvant anticancer therapy [19]. 
There is still very little information on the possible importance of CD71 in childhood acute lymphoblastic leukemias (ALL), and the data that is available is conflicting [17, 20, 21].

The aim of this study was to evaluate CD71 expression on acute lymphoblastic leukemia cells and to follow its possible clinical correlations.

\section{Material and methods}

Patients. Sixty one patients diagnosed with ALL in the Department of Pediatrics, Hematology, Oncology and Endocrinology, Medical University of Gdansk, from whom a surplus bone marrow (BM) was available after the diagnostic biopsy, were enrolled into this study between 2005 and 2009. The only inclusion criteria were diagnosis of acute lymphoblastic leukemia and material availability.

There were 27 girls and 34 boys, median age 5.3 years (from 1 to 17.6 years); with no significant differences between the genders. The bone marrows were routinely assessed by the hematological diagnostic laboratory and the leukemic blasts were classified according to their morphology (following French-American-British classification: L1-L3) and immunophenotype.

55 patients were diagnosed with B-lineage ALL, five patients had T-lineage ALL, and one patient biphenotypic T/B ALL. Immunophenotyping: 45 (74\%) patients had common ALL, three (5\%) proB, three (5\%) prepreB, two (3\%) preB, two (3\%) mature B-ALL, four (6.5\%) T-cell ALL, and one $(1.5 \%)$ preT ALL.

All BMs were cultured in order to obtain karyotype; chromosomes were obtained in 38 patients (62\%) and 29 were found to have chromosomal abnormalities, including 17 cases of hyperdiploidy and 12 cases of structural abnormalities. Bcr-abl mutation was investigated by fluorescence in-situ hybridization (FISH) in 49 patients, and was found positive in three.

Clinical data was retrospectively obtained from the routine clinical evaluations. Risk group qualification was based on the ALLIC 2000 criteria and was performed after 33 days of treatment. Patients were categorized as standard (SR), intermediate (IR), or high risk (HR). Patients who had died before the risk group assessment were, for the purposes of statistical analyses, allotted to the high risk group.

Organ involvement. Central nervous system involvement was defined as a presence of blastic cells in the cerebrospinal fluid, infiltration observed in the magnetic resonance imaging (MRI) scan, or clinical features of the cranial nerves involvement. Liver and spleen enlargement were clinically defined as organ margin palpable more than $1 \mathrm{~cm}$ below the appropriate costal margin.

Hematological abnormalities. Initial peripheral blood (PB) morphology was routinely evaluated with a Sysmex XE 2100 analyzer. White blood cell (WBC), hemoglobin (Hgb) and platelet (PLT) count were analyzed. Hyperleukocytosis was defined as PB white blood cell count (WBC) $>50,000 / \mu 1$.

Biochemistry. Initial PB serum uric acid (UA) and lactic dehydrogenase (LDH) were measured.

Treatment response. Treatment response was evaluated according to the ALLIC 2000 protocol: initial steroid response was evaluated based on the absolute count of blastic cells per $\mu \mathrm{l}$ in the $\mathrm{PB}$ on the $8^{\text {th }}$ day of the treatment protocol (Day 8 blastosis). Induction therapy response was assessed by microscopic analysis of the bone marrow specimens collected on the $15^{\text {th }}$ and $33^{\text {rd }}$ days of the chemotherapy protocol (respectively Day 15 BM and Day $33 \mathrm{BM})$. Remission pattern was denoted M1 (< $5 \%$ blasts cells in the BM, complete remission), M2 (5-25\% blast cells, partial remission), or M3 (> 25\% blast cells, no remission).

Disease-free survival (DFS) and overall survival (OS). DFS was defined as survival without leukemia relapse; for OS, deaths from all causes were included. All clinical evaluations are summarized in Figure 1.

Sample preparation. BM was obtained by aspiration biopsy; preservative free heparin was used as an anticoagulant. All biopsies were performed prior to treatment implementation. Bone marrow was first immunophenotyped to select markers for further blastic cells' staining. Mononuclear cells were isolated by density gradient centrifugation on Histopaque 1077 (Sigma-Aldrich). Interphase cells were collected and washed twice in RPMI medium (Sigma-Aldrich). Aliquots of $0.3 \times 10^{6} \mathrm{cells} / 50 \mu \mathrm{l}$ were used for further staining. From the separation onwards, all staining/washing steps were performed at $4^{\circ} \mathrm{C}$.

Antigen expression. CD71 surface expression was measured on bone marrow blastic cells. Staining to identify blastic cells (either CD19/CD10 or CD19/CD34 for B-lineage ALLs or CD7/CD34 for T-lineage ALLs) was chosen based on the routine bone marrow immunophenotyping performed for diagnostic purposes. The percentage of blastic cells in our bone marrow samples varied from $45 \%$ to $100 \%$ with a median of $90 \%$. Bone marrow mononuclear cells were stained with the following antibody conjugates: anti-CD71 FITC/anti-CD19 (or anti-CD10) PE/anti-CD34 (or anti-CD19) PE-Cy5 or anti-CD71 FITC/anti-CD7 PE/anti-CD34 PE-Cy5 (all antibodies were from BD Bioscience). $5 \mu$ l of each monoclonal antibody was added to the appropriate tubes and incubated for $30 \mathrm{~min}$. Finally, the cells were washed and analyzed by flow cytometry. Each sample was run with an appropriate isotype control. 
Extramedullary organ involvement

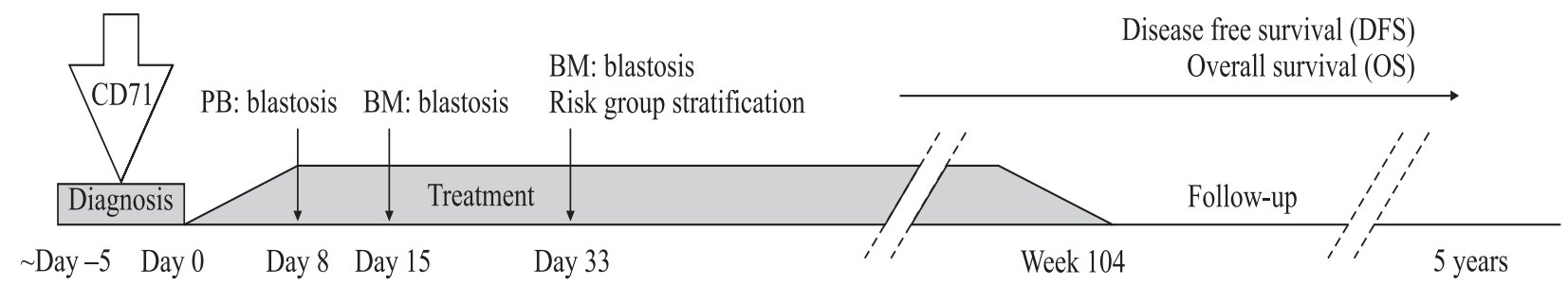

Figure 1. Schematic representation of the clinical evaluations performed in the study. CD71 flow cytometric measurement was performed at diagnosis, before treatment implementation
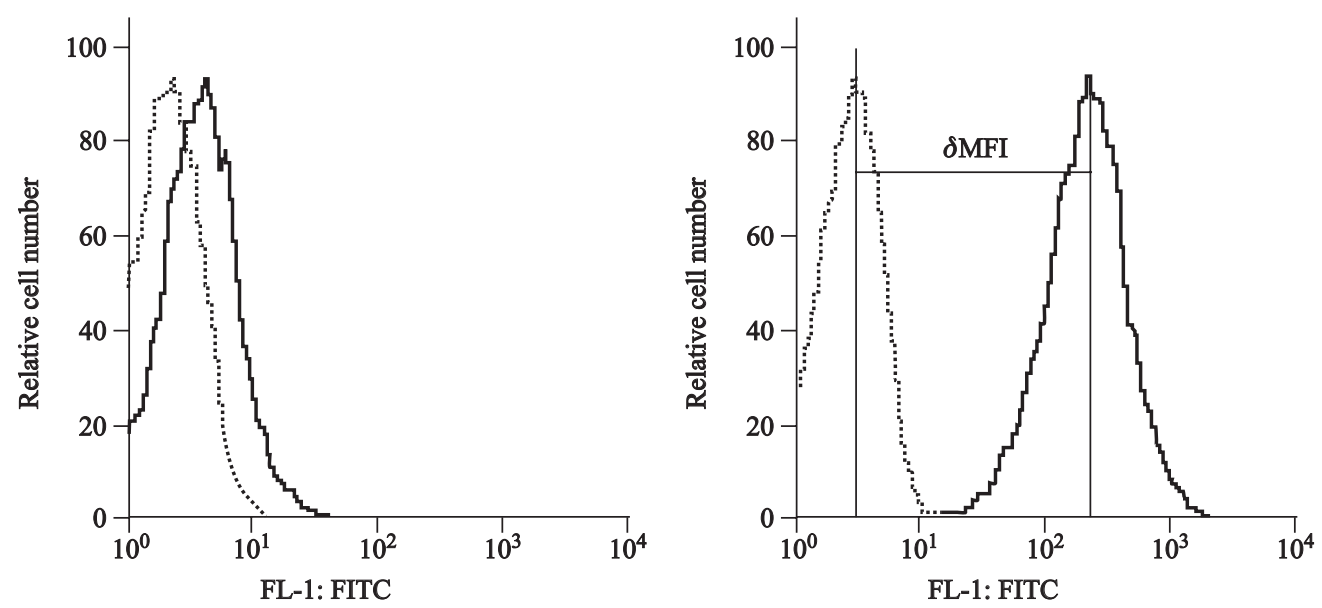

Figure 2. Examples of CD71 expression. Left panel: low CD71 expression on B-lineage ALL blasts. Right panel: high CD71 expression on T-lineage ALL. Isotypes are shown with dotted lines and anti-CD71 with solid lines

Flow cytometry analysis. Samples were analyzed by flow cytometry using a Becton Dickinson FACScan instrument equipped with a 488-nm argon laser. Five data parameters were assessed: linear forward and side scatter (FSC, SSC), FL-1(FITC), FL-2(PE) and FL-3 (PE-Cy5). CellQuest (Becton Dickinson) and FlowJo (ThreeStar) softwares were used for data analysis. Mean fluorescence intensity (MFI) was used as a measure of antigen surface expression. For individual samples, an anti-CD71 FITC $\delta$ MFI was calculated: $\delta \mathrm{MFI}=$ MFI of the population of interest - MFI of the appropriate isotype control (Figure 2). MFI is expressed in arbitrary values.

Statistical analysis. Statistica 8.0 PL (StatSoft) software was used for all of the analyses. The statistical significance of the results was analyzed by means of Mann-Whitney U test and Kolmogorow-Smirnoff. Spearman rank test was used to assess the correlation between the variables. Differences were considered of statistical significance if the $p$ value was $<0.05$. Disease free survival time and overall survival time measured from diagnosis were estimated according to the method of Kaplan-Meier and compared between groups by means of log-rank test.
The study was approved by the Local Ethics Committee; the patients and/or their parents (legal guardians) gave informed consent to participate in the study.

\section{Results}

CD 71 expression found on the leukemic blasts was diversified. In 56/61 patients (92\%), all blastic cells showed expression of CD71 with $\delta$ MFI median of 13 (ranging from 2 to 284), in four cases CD71 was found on a subpopulation of $17-24 \%$ blasts, only one leukaemia did not show expression of CD71 (Figure 2).

CD71 expression did not differ between sexes $\delta$ MFI median 10.6 for girls and $\delta$ MFI median 15.6 for boys, nor did it show a correlation with patient age $(\mathrm{Rs}=0.023)$.

\section{Immunophenotype}

CD71 expression was statistically higher on T-lineage leukemias, with $\delta$ MFI median for B-lineage ALL of 12.4 (range 2-100) and 27.9 for T-lineage ALLs (range 14-284) $(\mathrm{p}=0.01)($ Figure 3$)$. 


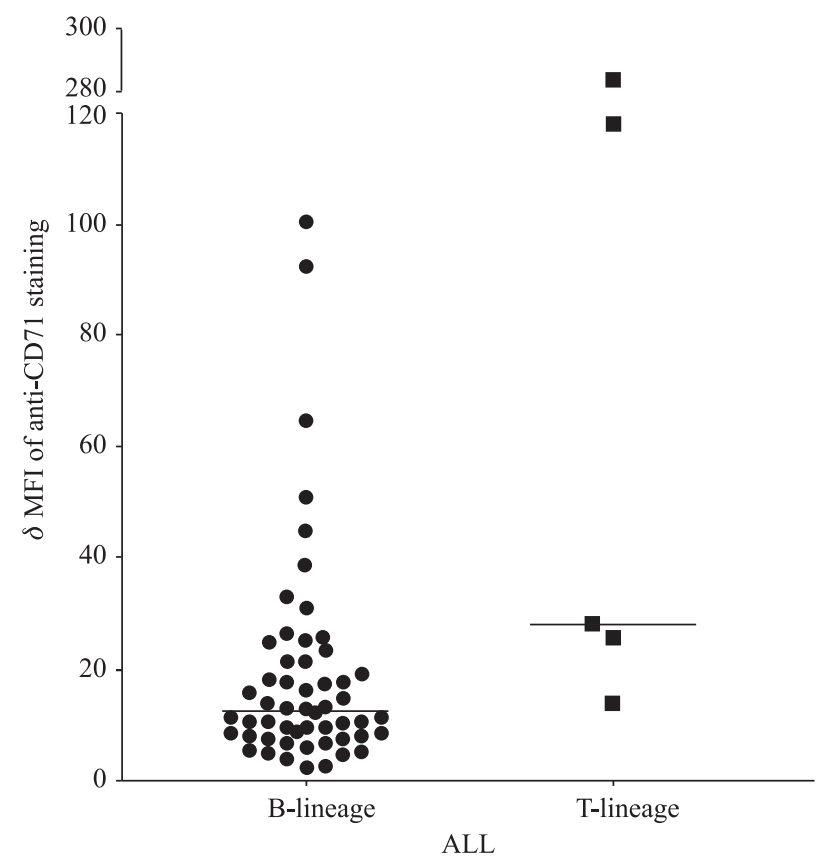

Figure 3. CD71 expression on the leukemic blasts assessed based on $\delta$ MFI of anti-CD71 staining. Horizontal lines indicate median values $(\mathrm{p}<0.05)$

Within the T-lineage group, the lowest MFI was noted in the only pre-T leukemia, while T-ALLs all showed higher MFIs. Within the B-lineage group, the highest MFI was seen on the mature B and proB blastic cells.

Within the B lineage ALL, a significant difference in CD71 expression existed between precursor B ALL and mature B-ALL showing higher CD71 expression levels $(p=0.02)($ Figure 4).

\section{Chromosomal abnormalities}

The presence of chromosomal abnormalities did not correlate with CD71 expression. Patients with chromosomal aberrations showed median CD71 expression of 15 (range 2-118) vs. 15.9 (range 6-284) in patients with normal karyotype of the leukemic blasts $(p=0.49)$. Hyperdiploidy was also not related to CD71 expression (median 10.5 in the hyperdiploidy group vs. 15.9 in non-hyperdiploid blasts; $\mathrm{p}=0.23$ ), nor was bcr-abl presence (32.1 vs. 13.6; $\mathrm{p}=0.17$ ).

\section{Initial laboratory and clinical parameters; initial organ involvement}

Patients with initial hyperleukocytosis $(\mathrm{n}=11)$ showed markedly higher CD71 expression on the blastic cells with median $\delta \mathrm{MFI}=25.7$ compared to the non-hyperleukocytosis patients $(\mathrm{n}=50)$ with $\delta \mathrm{MFI}=$

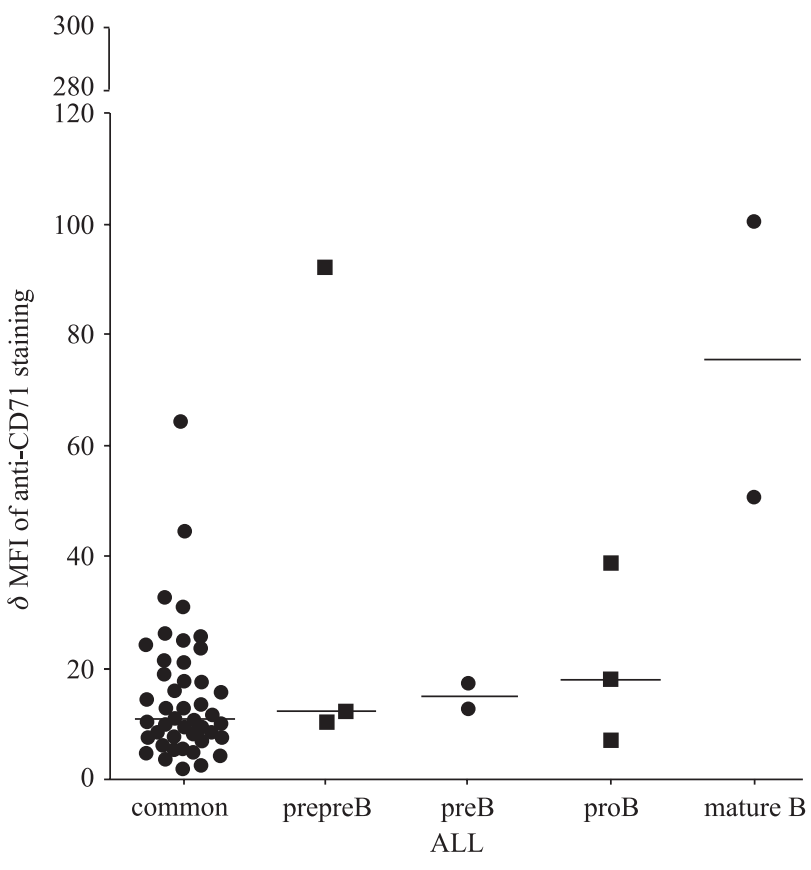

Figure 4. CD71 expression on B-lineage leukemic blasts assessed based on $\delta$ MFI of anti-CD71 staining. Horizontal lines indicate median values

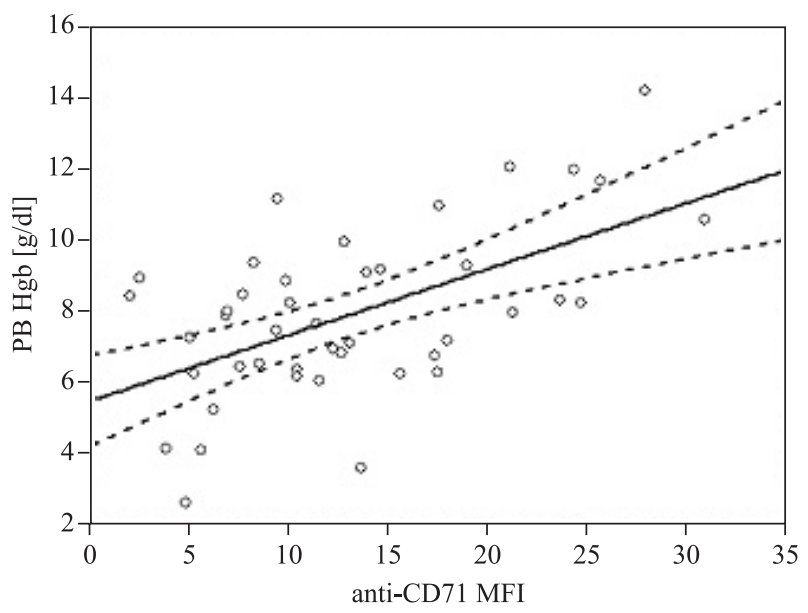

Figure 5. Correlation between peripheral blood hemoglobin concentrations (PB Hgb) and blastic CD71 expression in a group of patients with anti-CD71 MFI $<35$ $(\mathrm{n}=53, \mathrm{Rs}=0.38)$

$=11.8(\mathrm{p}=0.007336)$, although it was due to the prevalence of hyperleukocytosis in T-lineage leukemias. When only B-lineage ALLs were analyzed, the difference, although present $(\delta \mathrm{MFI}=11.5$ for patients with hyperleukocytosis $[n=6]$ and 15.4 for the remaining patients), was not statistically significant $(p=0.26)$.

$\delta$ MFI CD71 positively correlated with Hgb concentration at diagnosis $(\mathrm{Rs}=0.31)$, PLT count 
Table 1. Median MFI for anti-CD71 FITC staining (min-max) in groups with or without organ involvement

\begin{tabular}{|c|c|c|c|c|}
\hline \multicolumn{2}{|c|}{ Organ involvement } & CNS3 & CNS1 & p \\
\hline \multirow{2}{*}{ CNS } & Median MFI (min-max) & $13(2-283)$ & $13.6(5-100)$ & 52 \\
\cline { 2 - 5 } & $\mathrm{n}$ & 7 & No-hepatomegaly & \\
\hline \multirow{2}{*}{ Liver } & Median MFI (min-max) & $11.5(2-118)$ & $14.2(4-283)$ & NS \\
\cline { 2 - 5 } & $\mathrm{n}$ & 33 & 24 & NS \\
\hline \multirow{2}{*}{ Spleen } & Median MFI (min-max) & $16.5(2-118)$ & $11.9(4-283)$ & 29 \\
\cline { 2 - 5 }
\end{tabular}

Table 2. Median MFI for anti-CD71 FITC staining (min-max) depending on the treatment response

\begin{tabular}{|c|c|c|c|c|c|c|}
\hline & & \multicolumn{2}{|c|}{$>1,000$} & \multicolumn{2}{|c|}{$<1,000$} & $\mathbf{p}$ \\
\hline \multirow{2}{*}{8 day blastosis } & Median MFI (min-max) & \multicolumn{2}{|c|}{$12.9(7-283)$} & \multicolumn{2}{|c|}{$11.8(2-92)$} & \multirow{2}{*}{ NS } \\
\hline & $\mathrm{n}$ & \multicolumn{2}{|c|}{8} & \multicolumn{2}{|c|}{46} & \\
\hline & & M1 & M2 & M3 & Aplasia & \\
\hline \multirow{2}{*}{15 day BM } & Median MFI (min-max) & $10.6(4-33)$ & $18.9(6-92)$ & $12.9(2-283)$ & $13.6(4-64)$ & \multirow{2}{*}{ NS } \\
\hline & $\mathrm{n}$ & 19 & 7 & 10 & 19 & \\
\hline & & M1 & M2 & M3 & Aplasia & \\
\hline \multirow{2}{*}{33 day BM } & Median MFI (min-max) & $13.02(2-283)$ & & 32 & $11.2(4-23)$ & \multirow{2}{*}{ NS } \\
\hline & $\mathrm{n}$ & 41 & 0 & 1 & 12 & \\
\hline
\end{tabular}

$(\mathrm{Rs}=0.35)$ and with UA $(\mathrm{Rs}=0.30)$ and $\mathrm{LDH}(\mathrm{Rs}=$ $=0.26$ ) concentrations. Correlation with Hgb was stronger in the group of ALLs with lower CD71 expression $(\delta \mathrm{MFI}<35, \mathrm{n}=53)$ (Figure 5).

CD71 expression on the blastic cells did not differ between patients with extramedullary organ involvement (Table 1).

\section{Therapy response and risk groups}

CD71 expression did not differ statistically between the clinically evaluated risk groups. Therapy response was assessed based on several parameters: initial response to the steroid therapy expressed as a PB blastosis on the $8^{\text {th }}$ day of treatment, bone marrow analysis on the $15^{\text {th }}$ and $33^{\text {rd }}$ day of treatment. None of these parameters showed correlation with CD71 expression (Table 2).

\section{Disease-free survival (DFS) and overall survival (OS)}

There were no relapses or deaths in the T-lineage ALL groups. The B-lineage group was divided into two groups based on the MFI expression $-\mathrm{MFI}<$ median (i.e. MFI < 13) and MFI > median (MFI > 13). The 5 year DFS and 5 year OS probabilities were lower in the MFI > 13 group (DFS 0.64 vs. 0.91, Figure 6

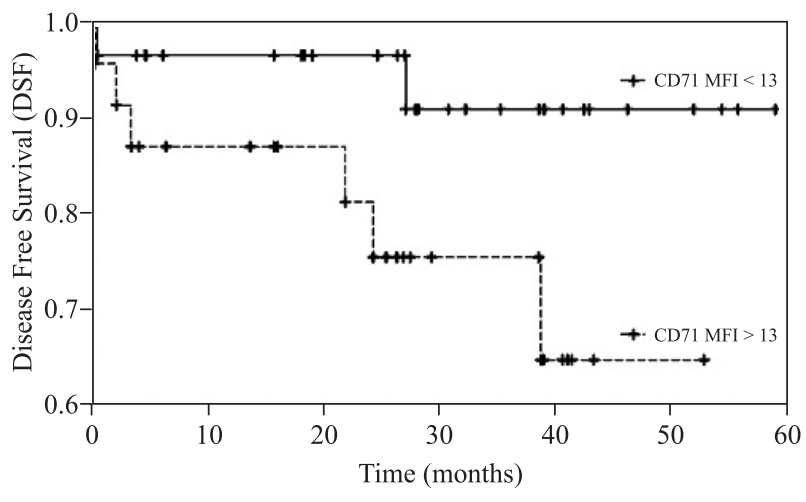

Figure 6. Kaplan-Meier curves presenting DFS in groups of B-lineage-ALL patients with phenotype: CD71 MFI $<13$ (continuous line) and CD71 MFI > 13 (dotted line)

and OS 0.81 vs. 0.97 , Figure 7) The mortality rate in the B-lineage ALL with CD71 MFI < 13 was $4 \%$ (1/25), while in the MFI > 13 group 20\% (4/20) patients had died by the end of the observation time.

\section{Discussion}

In normal human tissues, CD71 is found predominantly in the basal epidermis, endocrine pancreas, hepatocytes, Kupffer cells, testes, and the pituitary 


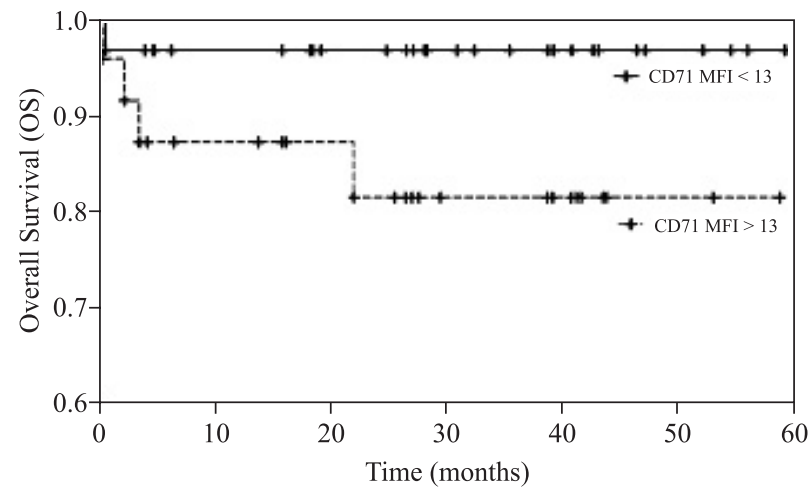

Figure 7. Kaplan-Meier curves presenting OS in groups of B-lineage-ALL patients with phenotype: CD71 MFI $<13$ (continuous line) and CD71 MFI > 13 (dotted line)

gland; it has also been detected on activated lymphocytes, natural killer cells, monocytes and macrophages [15, 22-25]. In hematopoiesis, CD71 is not present on a pluripotent stem cell, next it is detectable on erythroid progenitors, and again it is not detected on mature cells of the erythroid line [26]. Myeloid line bone marrow precursors are variably CD71 positive: dividing cells on the level of blast to myelocytes are all CD71 positive, while non-dividing late precursors (from metamyelocytes) are predominantly CD71 negative [26-28]. Quiescent PB lymphocytes, of both $\mathrm{T}$ and $\mathrm{B}$ lineages, lack CD71 which is produced in them after stimulation [2].

In contrast to a limited pattern of expression in normal tissues, CD71 is reported to be widely present on neoplastic cells $[2,15]$. Yeh et al. found increased transferrin binding in peripheral blood mononuclear cells from patients with leukemia, lymphoma and myeloma [29]. In B-cell chronic lymphocytic leukemia, CD71 expression is heterogenous, with some cases showing high expression [30-32]. Both B and $\mathrm{T}$ lineage non-Hodgkin's lymphomas have been reported to express CD71, with the expression level correlating with the lymphoma grading, though not with patient survival $[18,33]$. Transferrin receptor is overexpressed in adult T-cell leukemia, a neoplasm related to human T-lymphotropic virus type 1 (HTLV-1) with a poor prognosis $[34,35]$. It is also overexpressed in acute myeloid leukemia [14]. Investigation of CD71 expression and importance, although ongoing in many adult malignancies, did not draw much attention in childhood leukemias.

ALL in children has thus far been evaluated in terms of CD71 expression by two authors only [21, 17]. Koehler et al. investigated CD71 expression in childhood ALL by flow cytometric immunophenotyping, and found that $92 \%$ of T-lineage ALLs and 32\% of B-lineage ALLs were CD71 positive [21]. Das
Gupta et al. performed an immunohistochemical study of CD71 in ALLs and showed 62\% of T-lineage ALLs were CD71 positive, compared to $10.9 \%$ of positive common ALLs [17]. One more paper addressed CD71 expression in ex vivo ALL specimens from adults [20]. Glasova et al. reported CD71 expression on a few common ALLs with less than $10 \%$ of positive cells in the PB. Ten cases of T-ALL analyzed in this study showed a higher percentage of CD71 positive cells: from $13 \%$ to $75 \%$ [20].

Our study showed CD71 is present on blastic cells of almost all ALL cases, the expression being uniform within one case but significantly varied between cases. T-lineage ALLs showed higher CD71 expression than B-lineage leukemias, which is in concordance with the previously published data. Surface CD71 expression has been proven to be a downstream marker of Notch mediated PDK1 activation [20, 36, 37] and further proposed as a downstream marker of mTOR activity [38]. It seems that activity of survival pathway PI3K/PDK1/Akt/mTOR is linked with CD71 expression. In this context, high expression of CD71 on T-cell malignancies is not surprising as the importance of Notch signaling for T cell development has been well established [37]. Gain of function mutations of NOTCH $[39,40]$ as well as inactivating mutations of the suppressor PTEN and increased activity of PI3K/Akt pathway have been frequently identified in T cell ALL [41].

We demonstrated CD71 expression on most B-lineage ALLs including common ALL. Other authors report low frequency of CD71 positives in common ALL. This discrepancy could be explained by a different analysis approach whereby leukemias were classified as CD71 positive or negative only. Analysis of mean florescence intensity of CD71 staining on blastic cells allowed for identification of patients expressing smaller amounts of TfR1, which would be excluded by standard positive-negative analysis (Figure 2) [42]. Analysis of CD71 as a quantitative feature reveals more correlations with clinical data, such as $\mathrm{Hgb}$ and PLT. Like Das Gupta et al., we also found correlation of CD71 with initial hyperleukocytosis, but it was due to high frequency of hyperleukocytosis in T-lineage ALLs. The group analyzed by Das Gupta contained a relatively higher proportion of T-ALL cases, which is characteristic for the Indian subcontinent. The involvement of Notch and PI3K/Akt signaling in the development of some B-lineage acute lymphoblastic leukemia cases is emerging in the literature [39, 40, 43]. Diversity of CD71 expression among lymphoblastic leukemias originating from the B-lineage observed in this study might indicate different levels of PI3K/Akt/mTOR pathway activity in this group [44]. 
We also attempted an analysis of CD71 putative prognostic value in precursor-B ALL and found less favorable outcomes in patients with higher CD71 expression. Though the observation did not reach statistical significance, taking into account a relatively small number of events in our group and a short time of the follow-up, it seems noteworthy. Kohler et al. showed no relation between CD71 positivity and the EFS in a large group of patients. It is difficult to compare our data with Koehler's, as two decades ago EFS oscillated around $70 \%$ after three years, while in our material in the low CD71 group it exceeds $90 \%$ in a five-year perspective. Different protocols, improved supportive therapy, and improved outcomes of bone marrow transplantations have improved survival rates in all centers, making direct comparisons between old and new data impossible. Our group consisted of all the patients with ALL from whom bone marrow specimens were available over a period of four years in a single center with the same treatment protocol. The number of events noted allowed for statistical EFS and OS analysis only for B-lineage ALLs. We are continuing the follow-up of the patients included in this study.

In recent years, increasing effort has been focused in pediatric oncology on identifying molecular features of malignant cells in order to establish new risk factors, especially in the clinical low risk groups. The role of TfR1 requires further investigation in this context.

\section{Acknowledgements}

This study was supported by Polish Ministry of Science and Higher Education grant No 2 P05E 10330.

\section{References}

1. Aisen P. Transferrin receptor 1. Int J Biochem Cell Biol. 2004:36:2137-2143.

2. Ponka P, Lok CN. The transferrin receptor: role in health and disease. Int J Biochem Cell Biol. 1999;31:1111-1137.

3. Daniels TR, Delgado T, Helguera G, Penichet ML. The transferrin receptor part II: targeted delivery of therapeutic agents into cancer cells. Clin Immunol. 2006;121:159-176.

4. Daniels TR, Delgado T, Rodriguez JA, Helguera G, Penichet ML. The transferrin receptor part I: biology and targeting with cytotoxic antibodies for the treatment of cancer. Clin Immunol. 2006;121:144-158.

5. Sutherland R, Delia D, Schneider C, Newman R, Kemshead J, Greaves M. Ubiquitous cell-surface glycoprotein on tumor cells is proliferation-associated receptor for transferrin. Proc Natl Acad Sci USA. 1981;78:4515-4519.

6. Kondo K, Noguchi M, Mukai K et al. Transferrin receptor expression in adenocarcinoma of the lung as a histopathologic indicator of prognosis. Chest. 1990;97:1367-1371.

7. Zborovskaya I, Gasparian A, Kitaeva M et al. Simultaneous detection of genetic and immunological markers in non-small cell lung cancer: prediction of metastatic potential of tumor. Clin Exp Metastasis. 1996;14:490-500.
8. Seymour GJ, Walsh MD, Lavin MF, Strutton G, Gardiner RA. Transferrin receptor expression by human bladder transitional cell carcinomas. Urol Res. 1987;15:341-344.

9. Prutki M, Poljak-Blazi M, Jakopovic M, Tomas D, Stipancic I, Zarkovic N. Altered iron metabolism, transferrin receptor 1 and ferritin in patients with colon cancer. Cancer Lett. 2006;238:188-196.

10. Ryschich E, Huszty G, Knaebel HP, Hartel M, Buchler MW, Schmidt J. Transferrin receptor is a marker of malignant phenotype in human pancreatic cancer and in neuroendocrine carcinoma of the pancreas. Eur J Cancer. 2004;40:1418-1422.

11. Shindelman JE, Ortmeyer AE, Sussman HH. Demonstration of the transferrin receptor in human breast cancer tissue. Potential marker for identifying dividing cells. Int J Cancer. 1981;27:329-334.

12. Habashy HO, Powe DG, Staka CG et al. Transferrin receptor (cd71) is a marker of poor prognosis in breast cancer and can predict response to tamoxifen. Breast Cancer Res Treat. 2010;119:283-293.

13. Recht L, Torres CO, Smith TW, Raso V, Griffin TW. Transferrin receptor in normal and neoplastic brain tissue: implications for brain-tumor immunotherapy. J Neurosurg. 1990;72:941-945.

14. Barnett D, Wilson GA, Lawrence AC, Buckley GA. Transferrin receptor expression in the leukaemias and lymphoproliferative disorders. Clin Lab Haematol. 1987;9:361-370.

15. Gatter KC, Brown G, Trowbridge IS, Woolston RE, Mason DY. Transferrin receptors in human tissues: their distribution and possible clinical relevance. J Clin Pathol. 1983;36:539-545.

16. Taetle R, Ralph S, Smedsrud S, Trowbridge I. Regulation of transferrin receptor expression in myeloid leukemia cells. Blood. 1987;70:852-859.

17. Das Gupta A, Patil J, Shah VI. Transferrin receptor expression by blast cells in acute lymphoblastic leukemia correlates with white cell count \& immunophenotype. Indian J Med Res. 1996;104:226-233.

18. Habeshaw JA, Lister TA, Stansfeld AG, Greaves MF. Correlation of transferrin receptor expression with histological class and outcome in non-Hodgkin lymphoma. Lancet. 1983;1:498-501.

19. Brooks D, Taylor C, Dos SB et al. Phase Ia trial of murine immunoglobulin a antitransferrin receptor antibody 42/6. Clin Cancer Res. 1995;1:1259-1265.

20. Glasova M, Konikova E, Stasakova J, Babusikova O. The relationship of hla-dr, cd38 and cd71 markers to activation, proliferation and differentiation of some human leukemia and lymphoma cells. Neoplasma. 1998;45:88-95.

21. Koehler M, Behm F, Hancock M, Pui CH. Expression of activation antigens cd 38 and cd 71 is not clinically important in childhood acute lymphoblastic leukemia. Leukemia. 1993;7:41-45.

22. Le NT, Richardson DR. The role of iron in cell cycle progression and the proliferation of neoplastic cells. Biochim Biophys Acta. 2002;1603:31-46.

23. Yu Y, Kovacevic Z, Richardson DR. Tuning cell cycle regulation with an iron key. Cell Cycle. 2007;6:1982-1994.

24. Engleman EG, Warnke R, Fox RI, Dilley J, Benike CJ, Levy R. Studies of a human $t$ lymphocyte antigen recognized by a monoclonal antibody. Proc Natl Acad Sci USA. 1981;78: 1791-1795

25. Warnke R, Levy R. Detection of $t$ and $b$ cell antigens hybridoma monoclonal antibodies: a biotin-avidin-horseradish peroxidase method. J Histochem Cytochem. 1980;28: 771-776.

26. Blair A, Hogge DE, Sutherland HJ. Most acute myeloid leukemia progenitor cells with long-term proliferative ability in vitro and in vivo have the phenotype $\mathrm{cd} 34(+) / \mathrm{cd} 71(-) / \mathrm{hla}-\mathrm{dr}-$. Blood. 1998;92:4325-4335. 
27. Brandt J, Baird N, Lu L, Srour E, Hoffman R. Characterization of a human hematopoietic progenitor cell capable of forming blast cell containing colonies in vitro. J Clin Invest. 1988;82:1017-1027.

28. Sieff C, Bicknell D, Caine G, Robinson J, Lam G, Greaves MF. Changes in cell surface antigen expression during hemopoietic differentiation. Blood. 1982;60:703-713.

29. Yeh CJ, Taylor CG, Faulk WP. Transferrin binding by peripheral blood mononuclear cells in human lymphomas, myelomas and leukemias. Vox Sang. 1984;46:217-223.

30. Barnett D, Wilson GA, Lawrence AC, Buckley GA. Proposed relation between expression of interleukin-2 and transferrin receptors in b lymphocyte chronic lymphocytic leukaemia. J Clin Pathol. 1987;40:814.

31. Loisel S, Andre PA, Golay J et al. Antitumour effects of single or combined monoclonal antibodies directed against membrane antigens expressed by human b cells leukaemia. Mol Cancer. 2011;10:42.

32. Smilevska T, Stamatopoulos K, Samara M et al. Transferrin receptor-1 and 2 expression in chronic lymphocytic leukemia. Leuk Res. 2006;30:183-189.

33. Medeiros LJ, Picker LJ, Horning SJ, Warnke RA. Transferrin receptor expression by non-Hodgkin's lymphomas. Correlation with morphologic grade and survival. Cancer. 1988;61:1844-1851.

34. Moura IC, Lepelletier Y, Arnulf B et al. A neutralizing monoclonal antibody (mab a24) directed against the transferrin receptor induces apoptosis of tumor $\mathrm{t}$ lymphocytes from atl patients. Blood. 2004;103:1838-1845.
35. Petrini M, Pelosi-Testa E, Sposi NM et al. Constitutive expression and abnormal glycosylation of transferrin receptor in acute t-cell leukemia. Cancer Res. 1989;49:6989$-6996$.

36. Androutsellis-Theotokis A, Leker RR, Soldner F et al. Notch signalling regulates stem cell numbers in vitro and in vivo. Nature. 2006;442:823-826.

37. Kelly AP, Finlay DK, Hinton HJ et al. Notch-induced t cell development requires phosphoinositide-dependent kinase 1. Embo J. 2007;26:3441-3450.

38. Zheng Y, Collins SL, Lutz MA et al. A role for mammalian target of rapamycin in regulating $t$ cell activation versus anergy. J Immunol. 2007;178:2163-2170.

39. Aster JC, Pear WS, Blacklow SC. Notch signaling in leukemia. Annu Rev Pathol. 2008;3:587-613.

40. Zweidler-Mckay PA. Notch signaling in pediatric malignancies. Curr Oncol Rep. 2008;10:459-468.

41. Gutierrez A, Sanda T, Grebliunaite R et al. High frequency of pten, pi3k, and akt abnormalities in t-cell acute lymphoblastic leukemia. Blood. 2009;114:647-650.

42. Wells A, Loken MR. Flow cytometric mean fluorescence intensity: the biophysics behind the number. Leuk Res. 2008;32:845-846.

43. Zweidler-Mckay PA, He Y, Xu L et al. Notch signaling is a potent inducer of growth arrest and apoptosis in a wide range of b-cell malignancies. Blood. 2005;106:3898-3906.

44. O'Donnell KA, Yu D, Zeller KI et al. Activation of transferrin receptor 1 by c-myc enhances cellular proliferation and tumorigenesis. Mol Cell Biol. 2006;26:2373-2386.

Submitted: 24 October, 2010 Accepted after reviews: 18 September, 2011 